\title{
Design of a novel horizontal impact machine for testing of concrete specimens
}

\author{
P. Máca, J. Zatloukal \& R. Sovják \\ Faculty of Civil Engineering, \\ Czech Technical University in Prague, Czech Republic
}

\begin{abstract}
A novel design approach of an impact testing apparatus for concrete and highperformance cementitious composite specimens is described in this paper. To date, various approaches have been adopted when measuring the behaviour of concrete under impact loading. Most of the research teams used machines based on a guided drop-weight falling down freely on a concrete specimen. This paper describes the development process of a novel machine that is based on the pendulum principle. This experimental setup allows a horizontal placement of a specimen which has several advantages, such as easy elimination of a so called double hit, easy access to the sample and free space for the sensor mounting. The machine in the current setup is used for testing beam specimens, but, due to its modular concept it is also possible to rearrange the setup to test slabs utilizing a proprietary load transformer. The preliminary experimental results are shown in this paper as well as a description of the data acquisition system and adopted method of data filtration.

Keywords: impact, horizontal loading, concrete, filtration.
\end{abstract}

\section{Introduction}

The response of concrete subjected to impact loading is one of the main interests of several military and civil applications. For instance, government buildings and other types of important infrastructure are threatened by terrorist attacks or by accidental loading, such as aircraft crush or gas explosions. For this reason there is an increased interest in testing concrete elements by using proper dynamic analysis. When it comes to impact testing of concrete, the main issues encountered by many researchers are insufficiently fast data acquisition systems, 
vibrations in the loading frame and resonant frequency of the sensors. These issues then lead to apparent energy losses and large variance of the results between the laboratories. The large variance of the results is also connected with different techniques used for studying mechanical properties of concrete and concrete structures under dynamic loading. For instance, several authors used modified Charpy impact test [1] to study impact behaviour of concrete in bending. The modified Charpy test is limited by size of the sample and weight of the impact pendulum. Another way how to assess impact-bending properties of concrete are methods based on a principle of weight falling down on the sample from specified height, so called drop tower tests [2-4]. The disadvantage of these methods is the fact that a so called double hit caused by rebound of the impactor can occur in case when the specimen is not fully broken by the first blow of the impactor. Concrete in compression or direct tension subjected to high strain rates is usually tested by utilization of split Hopkinson pressure bar (SHPB). The compression loading can be uniaxial [5-7] or biaxial. The biaxial method is still in development but promises good results $[8,9]$. The basic principle of the method is that the specimen is placed between two long aluminium bars and an impulse is developed by a pressure gun into one of the bars. Impulse transmission is monitored by strain gauges and the difference between the magnitude of the pressure wave before and after the destruction of the specimen is acquired. The main disadvantage of this method is the fact, that the specimens are relatively small, usually cylinders with a diameter of $50 \mathrm{~mm}$.

This paper describes a new machine that has been constructed specifically for dynamic testing of concrete. At the moment the machine is in the bending loading configuration but it is relatively easy to transform it into the slab testing setup. The instrumentation and construction process of the machine is described in the paper.

\section{Principle of the impact machine}

The required horizontal loading setup and space capacity of the laboratories of the Czech Technical University governed the design of the impact machine. The target impact kinetic energy was set to $2000 \mathrm{~J}$ in the initial design, resulting in machine capable of dropping a $50 \mathrm{~kg}$ impactor from a height of $4 \mathrm{~m}$. In general, for impact test machines, there are several possible setups that can be divided into two groups, according to the direction of impact i.e. horizontal and vertical impact. The setup presented in this paper utilizes horizontal impact direction with stationary specimen where the impactor is following a circular trajectory using guide elements. The schematic diagram of the pendulum machine is shown in Fig. 1. The energy of the impact can be varied by placing the hoist of the impactor in different positions along the left column. The impactor is shown in three possible positions-heights in Fig. 1. The specimen is placed near the bottom return point of the impactor trajectory. This setup has several advantages and disadvantages, compared to the more conventional vertical impact. The advantage is that it is easier to avoid double impact just by geometric arrangement. The specimen is placed little further on the impactor trajectory, and 
due to the circular shape, little above, i.e. the point of impact is not the least potential point on the trajectory. Once the impactor hits the specimen, it rebounds and tends to balance in the bottom-return point of trajectory, not hitting the specimen again.

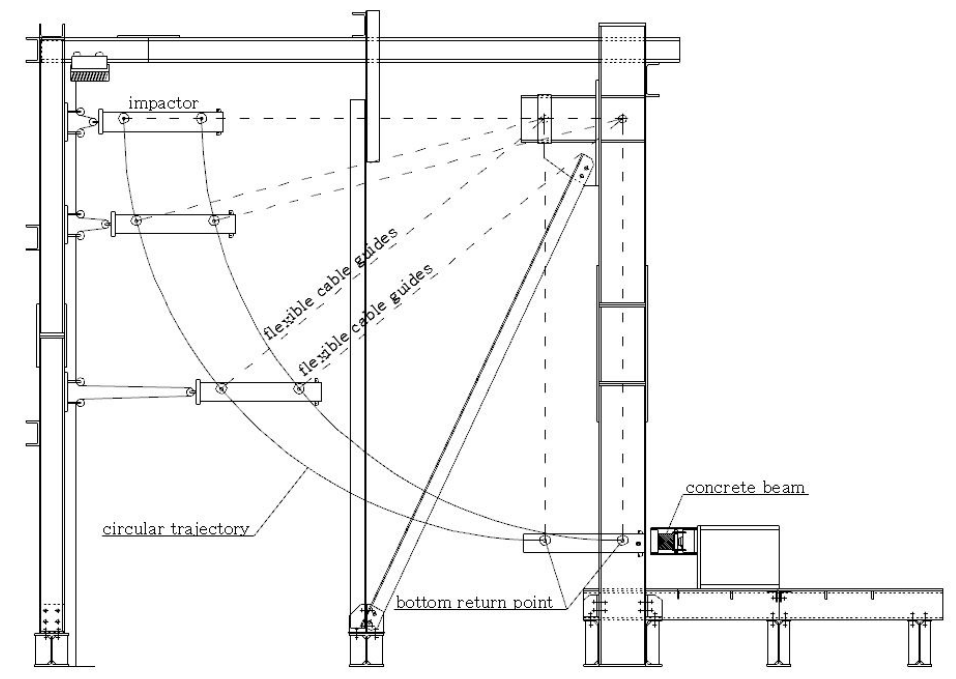

Figure 1: Schematic design of an impact pendulum machine. The impactor is shown in several possible launching positions.

The machine itself consists of several structural groups as shown in Fig. 1. These groups are the impactor support group, impactor guide group (Fig. 2) and specimen support group that is shown in detail in Fig. 3. These groups are highly modular and can be easily replaced. The impactor itself can be replaced as well. There were already five designs tested so far with different weights and crosssections from welded lightweight I-beams to solid rectangular steel bars. The impactor itself is instrumented with a set of accelerometers as shown in Fig. 2. It is possible to place the accelerometers to various positions along the impactor. The most interesting placement is near the tup of the impactor where four positions are available - top, bottom, right and left from the tup. It is also possible to place an accelerometer to the rear face of the impactor to measure the vibration of the impactor itself.

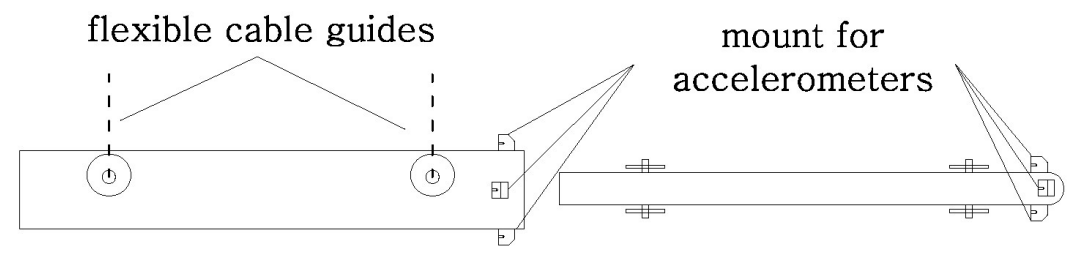

Figure 2: Location of accelerometers along the impactor. 
As long as the machine was intended as a prototype from the beginning, all parts are designed completely interchangeable and modular. It is possible to adjust the position of the axis of the impactor circular trajectory in $500 \mathrm{~mm}$ increments, from 1.5 to $3.0 \mathrm{~m}$ above the specimen position. Initially, the design was supposed to utilize drop height of double the axis height (with top point of trajectory above the axis level), using rigid guides. The rigid guides were designed as lightweight as possible, not to interfere with the impact mass, using duralumin profiles. During the testing of the machine, it was found out that the resonant frequency of the rigid guides was very near to the measured values during impact on concrete. For this reason it was impossible to filter this resonant frequency from the accelerations measured at the impactor tup and the design had to be changed. This change in design lead to replacing the rigid guide with flexible cable guides. Such solution smoothed out the acceleration readings, but resulted in the impossibility of dropping the impactor from heights above the circular trajectory axis.

The specimen itself is connected with steel yokes to the supports that are instrumented with load cells. The placement of the specimen is shown in detail in Fig. 3, the view is from above. The steel yokes allow the specimen to rotate freely along the support (as is shown in Fig. 4). This setup has an additional advantage, because it restrains the uplift of the specimen. At the moment the machine is prepared for measuring beams with a cross-section of $100 \times 100 \mathrm{~mm}$. The span of the beam can be from $300 \mathrm{~mm}$ to $1000 \mathrm{~mm}$.

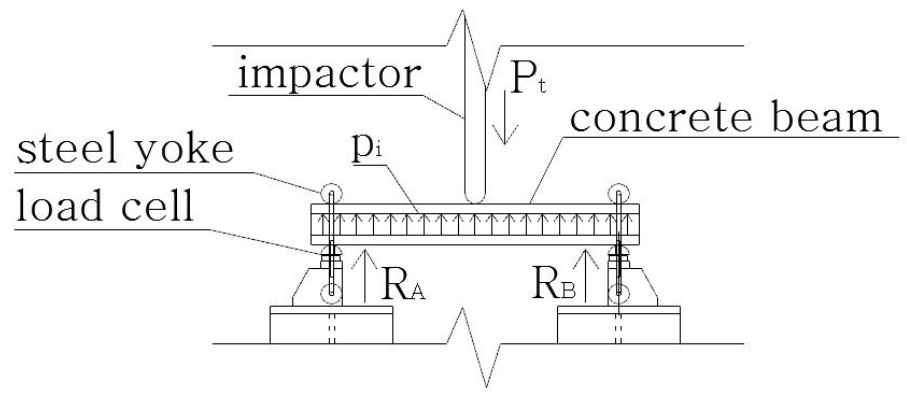

Figure 3: Specimen support group, top view.

\subsection{Measurement}

Proper dynamic analysis that takes into account inertial forces needs to be performed to adequately measure impact behaviour of larger concrete elements where high accelerations exist. There are several methods how to diminish the influence of the inertial forces. Sometimes an attenuator, usually made of plywood, is introduced in the contact zone between the loading impactor and the specimen $[10,11]$. To overcome the inertia, it is also possible to carry out a numerical analysis but this is fairly complex task. An experimental way how to deal with inertia was suggested in their work by Bentur et al. [12]. They proposed direct measurement of acceleration along the concrete specimen. In case a linear distribution of acceleration along the specimen is assumed, it is 
possible to calculate the acceleration at the mid-span of the specimen that is denoted $\ddot{u}_{o}(t)$. If the mass density $\rho$ and cross-sectional area $A$ remain constant throughout the length of the beam, it is possible to calculate the inertial load as:

$$
P_{i}(t)=\rho A \ddot{u}_{o}(t)\left[\frac{l}{3}+\frac{8}{3} \frac{h^{3}}{l^{2}}\right]
$$

where $P_{i}(t)$ is the inertial load at time $t, \rho$ is the mass density, $A$ is crosssectional area, $\ddot{u}_{o}(t)$ is acceleration at the mid-span, $l$ is the length of the midspan and $h$ is overhanging part of the beam.

The actual bending load $P_{b}(t)$ can be then obtained by subtracting the generalized inertial load $P_{i}(t)$ from the observed tup load $P_{t}(t)$ under the assumption of single degree of freedom system.

$$
P_{b}(t)=P_{t}(t)-P_{i}(t)
$$

However, in some cases such as highly fibre reinforced concrete as well as concrete with continuous steel reinforcement, the data obtained from the accelerometers mounted on the specimen are heavily influenced by reflected pulses and vibrations of the whole impact machine. For this reason an impact pendulum machine presented in this paper was instrumented with load cells under supports (Fig. 4) to measure the reaction forces. These reaction forces are not influenced by the inertia of the sample, while the load measured at the tup of the impactor contains the inertial forces of the specimen.
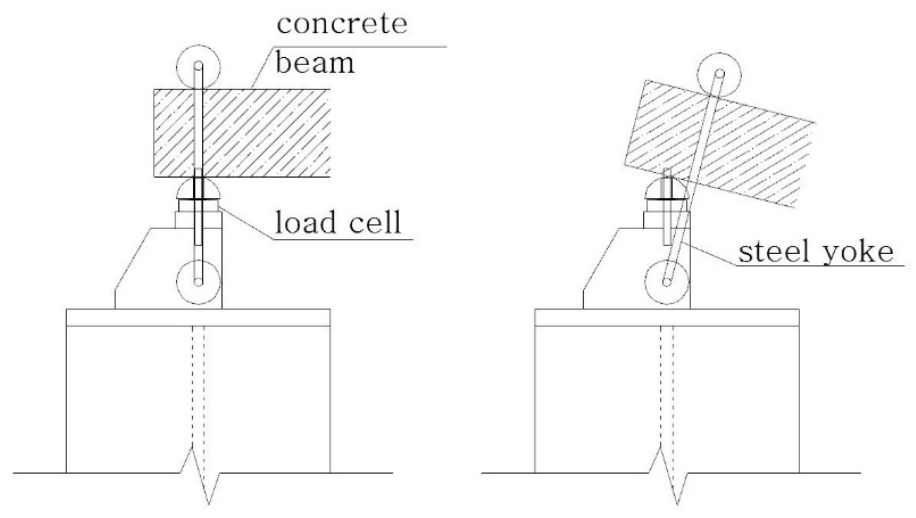

Figure 4: Instrumented supports that allow rotation of the sample and prevent an uplift of the specimen during an impact.

\subsection{Instrumentation used}

Measurement of dynamic properties of materials is quite complex problems and requires proper instrumentation. Inadequate data acquisition systems and low 
sampling rates can significantly influence the results. Two piezoelectric force sensors were used to measure the reaction forces between the support and the specimen as shown in Fig. 4. The load cells 200C50 were produced by PCB piezotronics and the maximal capacity of each load cell is $222 \mathrm{kN}$. Because these load cells are not capable of measuring tensile forces, the whole setup was prestressed to acquire these tensile forces. The acceleration of the specimen as well as accelerations of the impactor can be measured by up to five accelerometers $350 \mathrm{~B} 04$ produced by PCB with a maximal capacity of $50,000 \mathrm{~m} / \mathrm{s}^{2}$. These accelerometers can be placed into different locations as described above depending on the experimental setup. The resonant frequency of the accelerometers is higher than $100 \mathrm{kHz}$. The high resonant frequency of the instrumentation is particularly important because it can significantly influence the results.

The data acquisition system is triggered by two diffuse electric sensors OGT 500 that also allow measuring the accurate speed of the impactor and these are located at the lowest point of the impactor trajectory. The distance between the light gates is $200 \mathrm{~mm}$ and their switching frequency is $2 \mathrm{kHz}$. The gates were produced by IFM Electronic. The data acquisition system consists of a signal conditioner (483C05) produced by PCB with a frequency response higher than $1000 \mathrm{kHz}$. The signal conditioner decouples the AC signal from the DC bias voltage and provides constant current excitation to the PCB sensors. The decoupled signal is then acquired by a high precision analogue DAQ board ME5265 with 8 channels that can be sampled by rates up to $2 \mathrm{MHz}$ at 16 bit. During the experimental work it was found that sampling rates of $500 \mathrm{kHz}$ are adequate enough.

\section{Calibration of the machine}

During the calibration process of the machine a steel specimen was used. This allowed us to measure the response of the beam without the influence of fracture. Steel with yield strength of $235 \mathrm{MPa}$ and modulus of elasticity of $210 \mathrm{GPa}$ was used during this process. Impactor was dropped from the height of $0.3 \mathrm{~m}$ resulting in impact velocity equal to $2.42 \mathrm{~m} / \mathrm{s}$. The impact energy was $120 \mathrm{~J}$. Both reactions exhibited almost identical signal which indicated that the sample was hit in its centre point. The force measured by the load cells and the total reaction over time is shown in Fig. 5. Four accelerometers were mounted on the impactor's tup to measure the acceleration of the impactor during the impact process. As it is shown in Fig. 6 it is very complicated to read the acceleration on the impactor due to the high stiffness of the sample and the fact that high resonance occurred. The maximal measured acceleration during the first impact was $22000 \mathrm{~m} / \mathrm{s}^{2}$ and the first impulse duration was $0.15 \mathrm{~ms}$.

Because of the complicated data comparison an impact attenuator was used. The attenuator was made of $4 \mathrm{~mm}$ thick plywood and was placed in the contact area between the sample and the impactor. The measured impact has lower magnitude but is easily readable as is shown in Fig. 7. Maximal value was determined to be $3000 \mathrm{~m} / \mathrm{s}^{2}$ and the duration of the impulse was $2 \mathrm{~ms}$. 


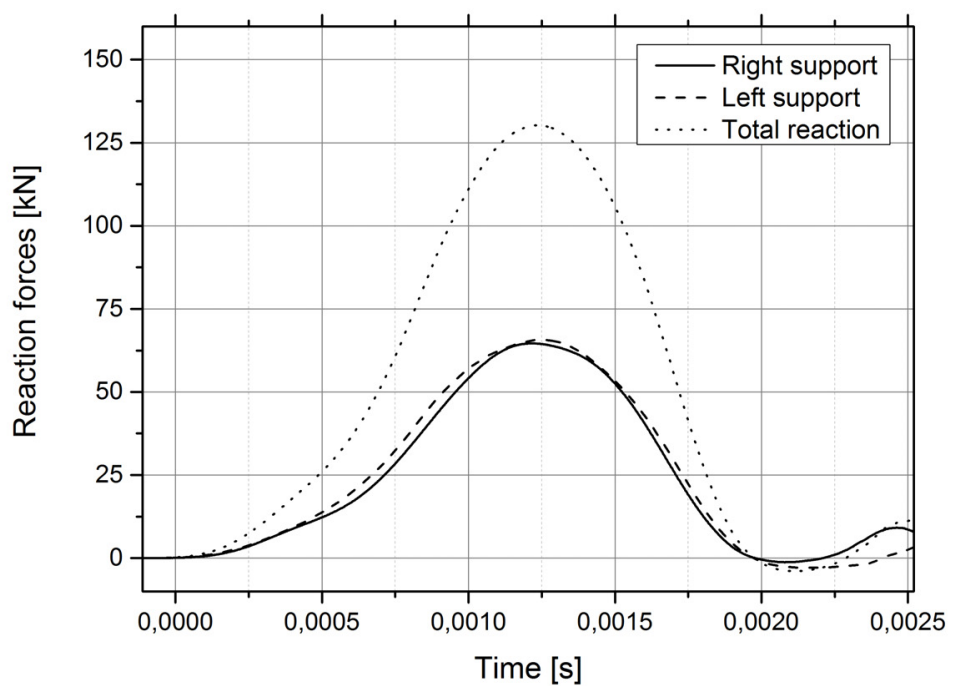

Figure 5: Response of the support reaction to impact loading of the concrete specimen.

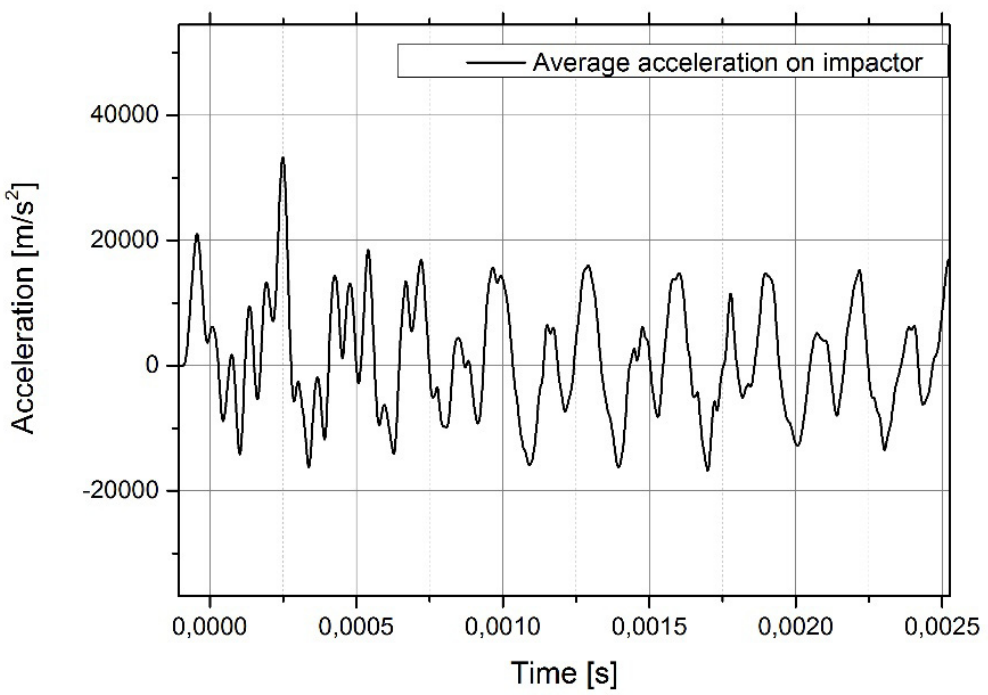

Figure 6: Response of the impactor to impact loading of steel specimen. 


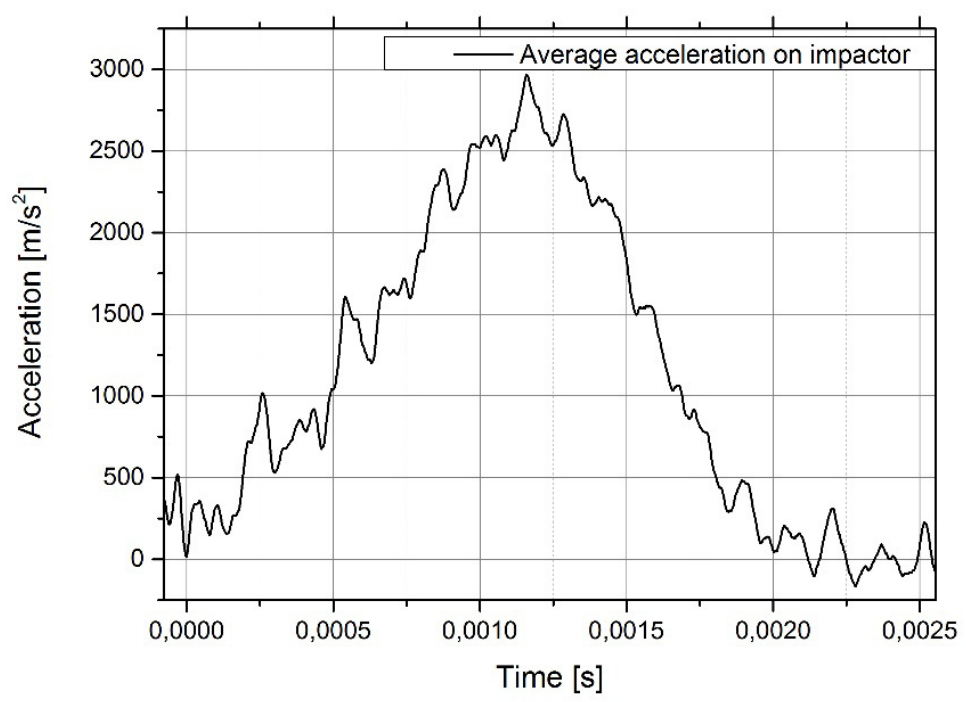

Figure 7: Response of the impactor to the impact usage of an attenuator.

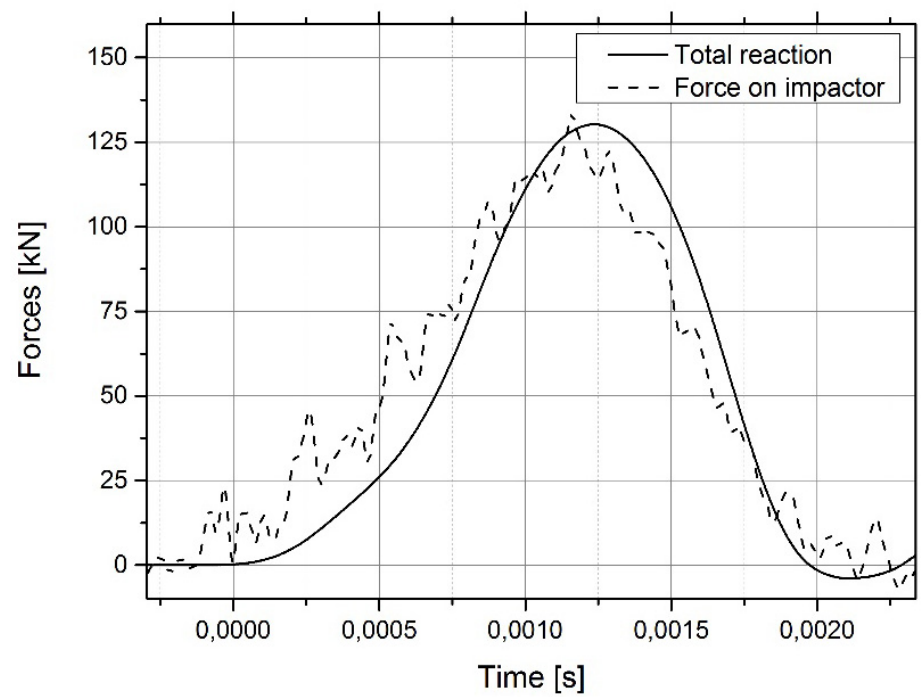

Figure 8: Response of the impactor to impact loading of the specimen compared to the support reactions with using attenuator.

Using Newton's second law of motion the force response gained from the impactor could be compared to the force signal gained from the load cells attached to the support reactions as is shown in Fig. 8. No signal filtering was 
used on the signal from accelerometers. In case a low pass parabolic $3 \mathrm{kHz}$ filter is used the signals nearly coincide. In the case of unfiltered signal a slight deviation between the signals can be seen as shown in Figure 8. The time difference between the maximal peek measured by the accelerometers on the impactor and the maximal force at the supports is caused by the length of the trajectory. The distance that the impulse wave needs to travel from the impact place to the reactions is approximately $0.3 \mathrm{~m}$.

\section{Conclusions}

A novel design for impact testing machine of concrete specimens was presented in this study. It was demonstrated that it is more convenient to have horizontal impact in order to avoid so called double hit and also to provide more comfortable space for instrumentation of the sample. Design of the impact machine is highly modular and it is very simple to change its configuration for testing the slabs. Steel yokes were successfully applied in order to avoid uplift of the specimen. After comparing several impact tests sampled by different rates, it was stated that $500 \mathrm{kHz}$ sampling frequency is sufficiently enough for measuring response of materials such steel or concrete even in case without any attenuator. In the case of attenuator usage, the sampling frequency can be as low as $10 \mathrm{kHz}$. It was also established that flexible cable guides should be provided for connection of the impactor to the rest of the machine in order to avoid eigen frequencies of the guide system similar to the eigen frequency of the sample. This behaviour would disturb the signal gained by the sensors attached to the sample. In the future research the authors plan to test different types of concrete under impact using the described machine. The loading strain rate will be varied by using an attenuator and different impact heights. This should lead to the deeper understanding of concrete behaviour under impact loading at intermediate strain rates, that correspond to falling rocks or vehicle impact.

\section{Acknowledgements}

The authors gratefully acknowledge the support provided by the Ministry of Interior of the Czech Republic under project number VG20102014003 on Experimental development of high-performance concrete with improved resistance against shock loading and high explosion energy absorption and dissipation capacity. The authors would also like to acknowledge the assistance given by the technical staff of the Experimental Centre, CTU in Prague and by students who participated in the project.

\section{References}

[1] Al-Oraimi, S. K., Seibi, A. C., Mechanical characterisation and impact behaviour of concrete reinforced with natural fibres. Composite Structures, 32, pp. 165-171, 1995. 
[2] Máca, P., Konvalinka, P., Curbach, M., Behaviour of Different Types of Concrete under Impact and Quasi-Static Loading. Applied Mechanics and Materials, 486, pp. 295-300, 2014.

[3] Zhang, X. X., Ruiz, G., Yu, R. C., A New Drop-Weight Impact Machine for Studying Fracture Processes in Structural Concrete. Strain, 46, pp. 252-257, 2010.

[4] Soleimani, S. M., Banthia, N., A Novel Drop Weight Impact Setup for Testing Reinforced Concrete Beams. Experimental Techniques, 37, 2012.

[5] Mechtcherine, V., Millon, O., Butler, M., Thoma, K., Mechanical behaviour of strain hardening cement-based composites under impact loading. Cement and Concrete Composites, 33, pp. 1-11, 2011.

[6] Wang, Z. L., Zhu, H. H., Wang, J. G., Repeated-Impact Response of Ultrashort Steel Fiber Reinforced Concrete. Experimental Techniques, 36, 2011.

[7] Quast, M., Hummeltenberg, A., Curbach, M., High Performance Lightweight Aggregate Concrete under Impact Loading. BetonStahlbetonbau, 107, pp. 15-22, 2012.

[8] Hummeltenberg, A., Curbach, M., Design and construction of a biaxial Split-Hopkinson-Bar. Beton-Stahlbetonbau, 107, pp. 394-400, 2012.

[9] Just, M., Quast, M., Curbach, M., Concrete at high dynamic biaxial loads. Performance, protection and strengthening of structures under extreme loading, The Indian concrete journal, 2013.

[10] Millard, S. G., Molyneaux, T. C. K., Barnett, S. J., Gao, X., Dynamic enhancement of blast-resistant ultra high performance fibre-reinforced concrete under flexural and shear loading. Int. J. Impact Eng., 37, pp. 405413, 2010.

[11] Habel, K., Gauvreau, P., Response of ultra-high performance fiber reinforced concrete (UHPFRC) to impact and static loading. Cement and Concrete Composites, 30, pp. 938-946, 2008.

[12] Bentur, A., Mindess, S., Banthia, N.: The behaviour of concrete under impact loading: Experimental procedures and method of analysis, Materials and Structures. 19 371-378. (1986). 